\title{
Research Advancements Towards in Existing Smart Metering over Smart Grid
}

\author{
Abdul Khadar A \\ Associate Professor \\ Dept. of E\&E Engg. \\ BITM Ballari, Karnataka, India
}

\author{
Javed Ahamed Khan \\ Professor \\ Dept. of E\&E Engg. \\ MIT Madanpalli, A.P., India
}

\author{
M S Nagaraj \\ Professor \& HOD \\ Dept. of E\&E Engg \\ BIET, Davangere, Karnataka, India
}

\begin{abstract}
The advent of smart meters has automated the entire process of billing generation system over commercial energy usage which was previously done using digital meter. Although western countries practice its usage more, it is still unknown to many developing countries along with its power distribution. Hence, this paper reviews the working principle of smart meters along with the brief of basic operation description. It thoroughly investigates the implementation work towards algorithm design and techniques developed that are being carried out in last five years towards smart meters. The paper examines the various significant technology that has evolved to address the problems in smart meter e.g. performance improvement, energy efficiency, security factor, etc. Finally, a set of research gap is explored after scrutinizing the advantages and limitations of existing techniques followed by brief highlights of the feasible line of research to compensate the unaddressed problems associated with research work direction towards smart meters.
\end{abstract}

Keywords-Digital Meter; Energy; Power Distribution; Performance; Privacy Smart Meter; Smart Grid

\section{INTRODUCTION}

There has been a huge revolution in the area of consumer electrical usage. In the existing system, a normal analogue meter is installed in the consumer house which keeps constant readings about the usage of the energy by the consumer. Such meters could be easily open, can be easily tampered, can be corrupted without even a trace [1]. However, all these malpractice negatively affects the economics due to faulty pricing and billing [2]. Therefore, in order to resist this, a smart meter comes as a boon to overcome such problems. Basically, a smart meter is a sophisticated meter which has both analogue and digital component and is installed in premises which needs to be billed for energy usage. Smart meter captures and records the frequency as well as voltage as the electrical data and highly supports communication in bidirectional pattern existing between the central and meter system. The data generated by the smart meter consists of timestamp information, identifier of unique meter, values of electricity consumption, etc. Smart meter also allows controlling the load remotely. It has also the capability to govern various utility devices in order to balance the load and demands. Different from conventional analogue meters, the readings from the smart meters are in digital form that is automatically sent to the suppliers by various communication means [3]. The recording of the energy usage from different premises are subjected to analysis and specific processing from the suppliers who then forwards the processed and highly well-structured report of energy usage to the customers. This report arrives at hand-held device in the form of graphical display which is quite a user friendly and very easily understands the usage statistics [4]. The consumer of conventional analogue meters has to wait for long period of due date to realise their usage statistics as well as their due amount. By that time, it is almost out of scope for a customer to save any electricity. But usage of smart meters allows dispensing the highly processed data about usage statistics to the customer in real time (i.e. 24/7). This lively report generates an awareness to save the electricity as well as money. Although, it is quite old concept in European nation, but in reality the usage of smart meters are far from reality in many developing countries like India. There are also many research impediments towards this field. The first biggest problem is to get an answer to a question: Can the smart meter performs energy efficiency? This is because smart meters really don't save any energy but it just makes customer aware about their usage scenario and the entire decision is left to the customers. The second big problem is to answer the question: How can wireless transmission assist in energy efficiency using smart meters? There are many studies where smart meters are found to use multiple wireless standards e.g. GSM, WLAN, XBee etc., but there are various hidden unanswered aspect like wireless networks are always error-prone to offer less QoS and less security. Such factors were not found to be addressed in any existing research manuscripts. The third challenging question is: How to ensure optimal security without impacting network performance? Usage of encryption algorithm over the large and massive bit streams of data generated from metered readings will invite delay and latency over the network and seriously affect the QoS performance. On the other hand, the usages of smart meters are more applicable in upcoming IoT applications [5] [6], which means various lethal threats travelling over internet will now attempt to compromise the metered readings. Hence, it requires thorough investigation about the existing solution and finding the best solution. Hence, this paper reviews all the significant and standard research papers and performs an exhaustive review of literature to scale an effectiveness of the existing techniques. Section 2 discusses about the essentials of smart meters followed by its management techniques in Section 3. The existing research in smart meters is elaborated in Section 4 with respect to advantage as well as limitation. It is then followed by research gap in Section 5. Possible line of research to circumvent the problems of existing system is briefed in Section 6 followed by conclusion in Section 7. 


\section{ESSENTIALS OF SMART METERS}

The smart meters can be defined as the electronic device that performs seamless monitoring and recording of the energy usage by digital means. Different from conventional metering system, where the readings have to be collected by the service providers, smart meters autonomously forward the readings to its service providers for impartial and error-free billing purpose. It has basically two simple components as shown in Figure 1. The basic smart meter is installed in the premises while the customer keeps the smart meter display device that is used for showing the usage statistics lively.
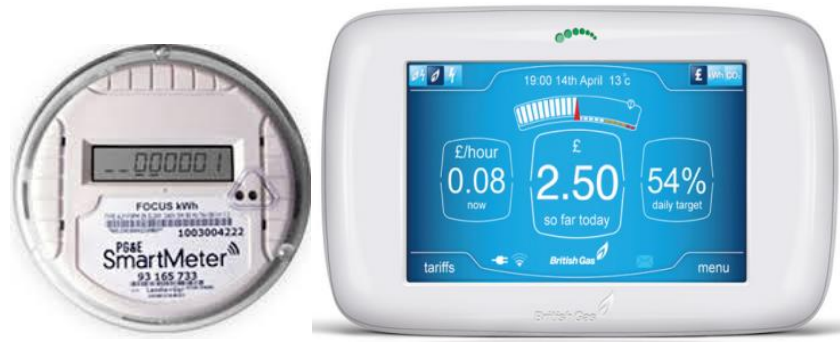

Fig. 1. a) Smart Meter b) Smart Meter Display Device

Usage of smart meters is quite important in next generation of technological advancement. Figure 2 is the direct demonstration of the usage of smart meter mechanism that shows the smart meter to capture the usage data from source point followed by transmission of data to service provider. Smart meters also make use of highly secured national communication network in order to involuntarily transmit the actual usage data to service providers [7]. The transmitted data area analysed to generate bill, whose information can be than directly accessed by the paid customers. The interesting fact here is a customer is always aware of their usage statistics and billing details and they don't have to wait for their due date to know about it. This awareness motivates and prompts the customer to save energy. There are multiple benefits of using smart meters, e.g.

- Error-free Bill Generation: The first and foremost advantage of smart metering system is generation of

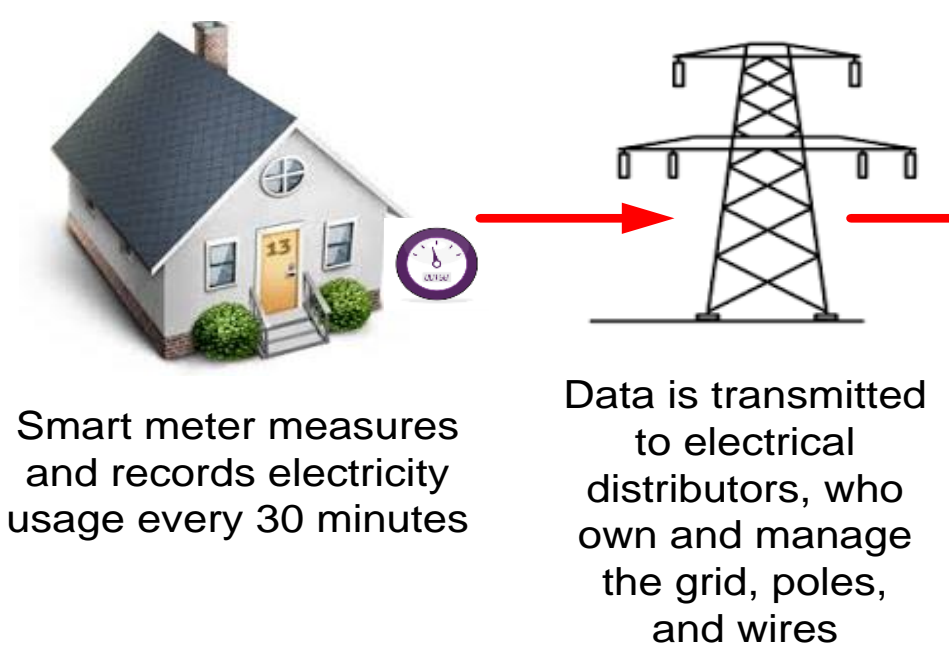

Fig. 2. Billing Mechanisms of Smart Meters highly accurate billing amount based on original usage data. Moreover, it allows the customer to track and record their usage 24/7; it doesn't give rise to any billing dispute and hence successfully maintains higher transparency in service usage.

- Awareness of Usage: Using smart meter display device, the customer can consistently track their usage. They can also make further strategy for energy conservation or fine tune their lifestyle accordingly. A better visibility of energy usage is enabled by the smart meter display device.

- Allows Faster Switching of Energy Suppliers: In developing countries like India, customers neither have any option nor has any idea about their service providers. Although number of service providers is very less, not even 10 because still it is analogue system which is quite traditional way. But with futuristic advancement with technologies, various service providers will be mushrooming offering competitive services with better charges. Hence, usage of smart meters allows the customers to switch to other service providers in a matter of minute.

- Supports Green Ecosystem: Smart meters are originally meant to work on a smart grid system that has higher supportability of minimal carbon emission and other green-house elements.

- Analytics on Usage Data: The usage data from smart meters are massive in size and hence attracts the area of analytics to extract more knowledge from the data. The service providers can use the analysed data to improvise their services.

An interesting part of smart meter is that it should be installed in the premises by the service providers at no cost. Usually, the roll out cost of the smart meter is meant to be covered up only in the billing system almost like the analogue metering system. Hence, installation, maintenance and aftermath of its usage is higher beneficial to customers as well as service providers.

\section{Energy providers make consumption data available via web-portal and in- home display}

\section{Monitor \\ household energy usage}




\section{OPERATIONS In SMART METER MANAGEMENT}

A smart meter consists of various sophisticated components, which includes i) a software system to perform usage data computation, ii) hardware system to support the digital reading with various electrical and electronic subdevices, and iii) a calibration mechanism that performs reading of the energy utilities. An essential building block of a conventional architecture of smart meter is shown in Figure 3. The common components available in smart meter systems are module for power management module, data communication, system-on-chip metering system, module for identifying any forms of tampering, clock that works on real time, supervisory module, voltage reference, transformer driver, etc. [8] [9]. The core backbone of the smart meter is basically system-on-chip processor along with core architecture supporting it. The differential inputs are supported by the analogue-to-digital converters in its front end [10]. The sensors with low-input receive its gains from integrated gain stage. Numerous intensive operations can be highly boosted using SoC chip with hardware multiplier while carrying out computation of energy. Various computation of power factor, voltage RMS current, reactive and active power, voltage, frequency, etc. are always on active process while smart meter is in operation. This section will discuss about the two essential components of the smart meters, i.e. analogue component and digital component.

\section{A. Analogue component of smart meter}

Basically, an analogue component is responsible for offering a hardware-bridge between two points where first point refers to generation of energy usage data and second point refers to software that processes the data and transmits it to service provider. The analogue component consists of Antialiasing filter, real-time clock, power supply, current and voltage measurement, sigma-delta analogue-to-digital converter, anti-tampering circuitry, battery charger, and harmonic analysis [11]. The anti-aliasing filter is used for filtering the spike using resistor, RC low pass filters, and voltage divider. Real-time clock is used to timestamp the utility data to show the time of capture. The power supply unit assists in supporting functions from the electrical mains or by using transformer. Current and voltage measurement is carried out using resistor while voltage is computed as drop across the current transformer and resistor. Analogue to digital converter is used to process current and voltage signal. Another essential unit is anti-tampering circuits which are directly connected with current sensors to identify any attempts of tampering the circuits. An analogue component also has a backup battery within it, which is used for charging the component during voltage in step-down stage. The component also protect itself from the transmission loss using harmonic elimination process from the analogue signals using Fourier-based techniques, band limiting filters, and adaptive real-time monitoring.

\section{B. Digital component of smart meter}

The digital component of the smart meter is supported by registers, microcontrollers, and RAM. The core object of digital component in smart meter is microcontroller as it performs all the computation, records and save value, carries out forwarding of utility data based on the standards of ANSI C 12.22 considering the data format to be ANSI C12.19 [12]. Smart meter uses Local Area Network (LAN) as well as Wide Area Network (WAN) in order to collect the usage data after a periodic interval of time. It also uses Power Line Carrier (PLC) and Radio Frequency (RF) in order to perform communication over grid interfaces. The different products of smart meters are briefly discussed in [13], which will show multiple operations carried out by different products of smart meters available in commercial market. Advanced Metering Infrastructure (AMI) is deployed over smart grid for enabling the process of aggregating the data from smart meters. Apart from these smart meters also adheres to standards e.g. ANSI CI2.19, ANSI C12.22, C12.19, [12].

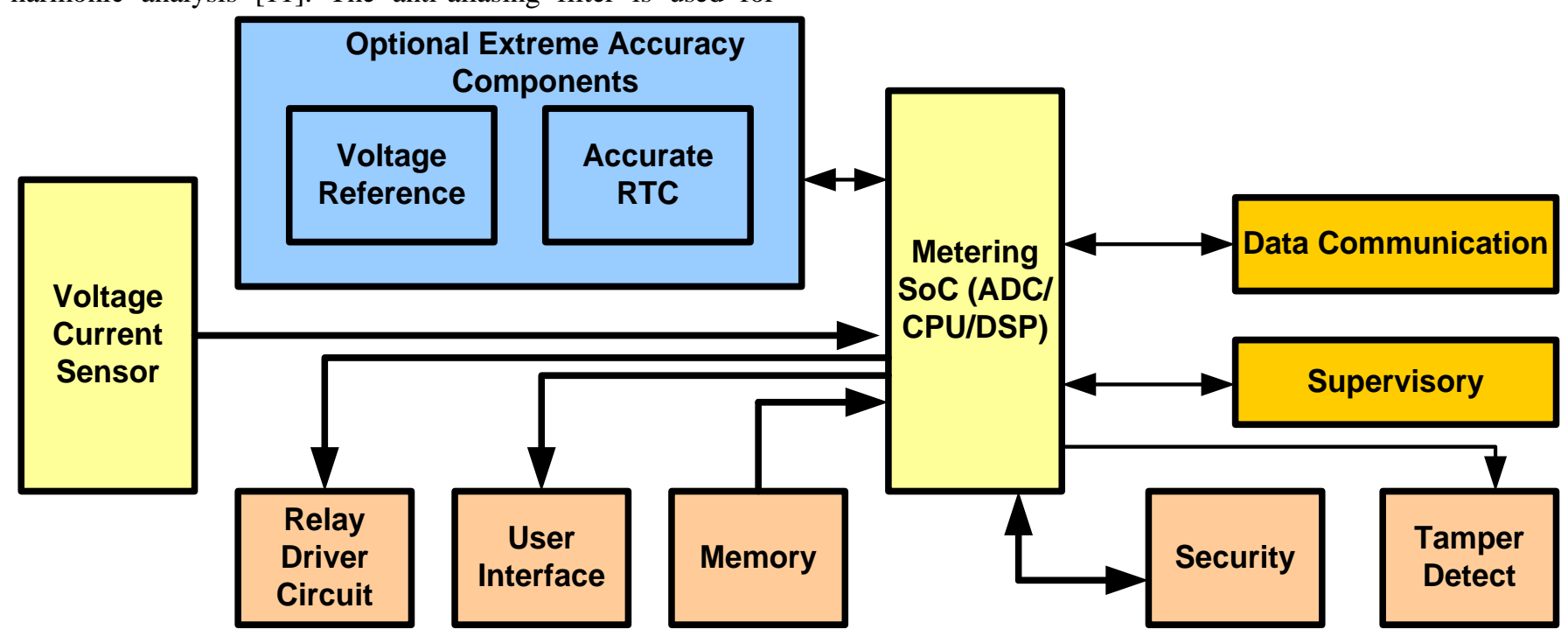

Fig. 3. Smart Meter Architecture

\section{EXISTING RESEARCH IN SMART METERING}

The research works toward addressing the problems of smart meters and enhancing its performance dates back to 1986 by Arthur H. Rosenfeld [14]. At present there are roughly 3,670 research manuscript with IEEE Xplore based on smart meters. At present there are various existing review papers e.g. 
[15]-[20] that have already discussed the frequently adopted approach but didn't discuss the advantages or limitations of it. The contribution of our work will be to present a thorough survey of recent techniques and measure their effectiveness. Hence, we will be discussing only the paper published between 2010 and 2016 that will act as an update to prior review work. This section will discuss about various techniques implemented by different researchers towards improving the efficiency of smart meters.

\section{A. Techniques towards performance enhancement}

One of the biggest challenging factors of Smart meter is to determine its state. Owing to unsynchronised form of signals and lag of time between the readings, the availability of network of smart meter cannot be guaranteed. Alimardani et al. [21] [22] has addressed this issue by developing a system that can evaluate the error variance of signals as a means of compensating the signals that are not synchronised. The authors has used IEEE 13-bus system and assessed its result using load distribution and error values over time. Towards performance enhancement, the work carried out by Ciuciu et al. [23] was claimed to enhance multi-dimensional features of smart metering performance e.g. security, identifying meters with equivalent objectives, data exchange, energy-demand management. The author has also presented an architecture (Figure 4) who has client layer integrated with security management and the top two layers are further integrated with the smart device layer using middleware.

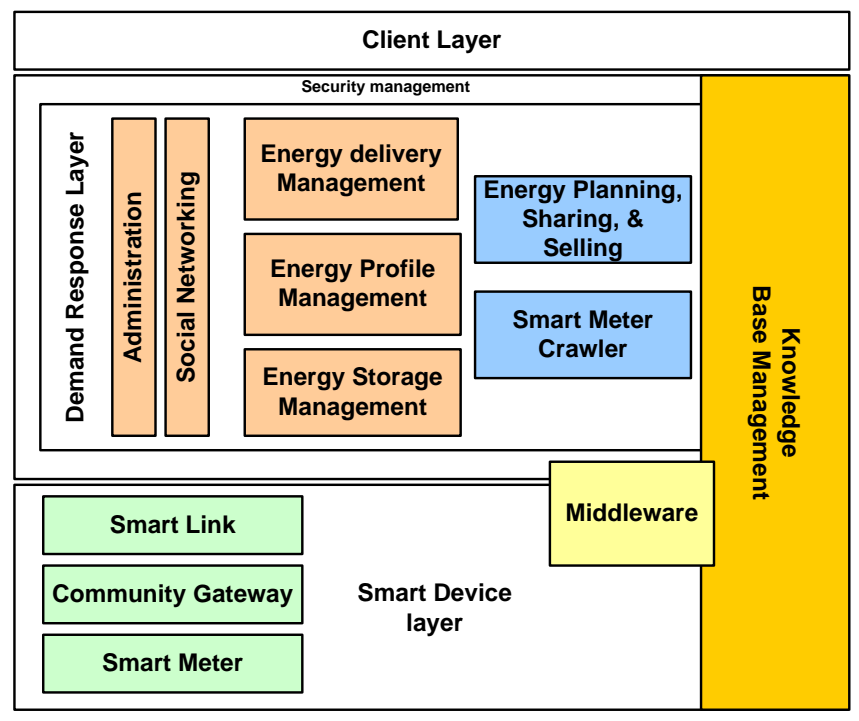

Fig. 4. Technique presented by Ciuciu et al. [23]

Most recently, the work carried out by Dede et al. [24] has presented a technique that designs the smart meter in the form of sensor network. In this work, the author has discussed about architecture of smart meter exclusively designed for future technologies with respect to sensor network (Figure 5). The architecture shows Elaboration Block (Elab), Analogue-toDigital Block (ADC), and communication block (Comm). The authors have developed an experimental test-bed in order to validate their prototype. The study outcome was found to possess less than $5 \%$ of voltage.

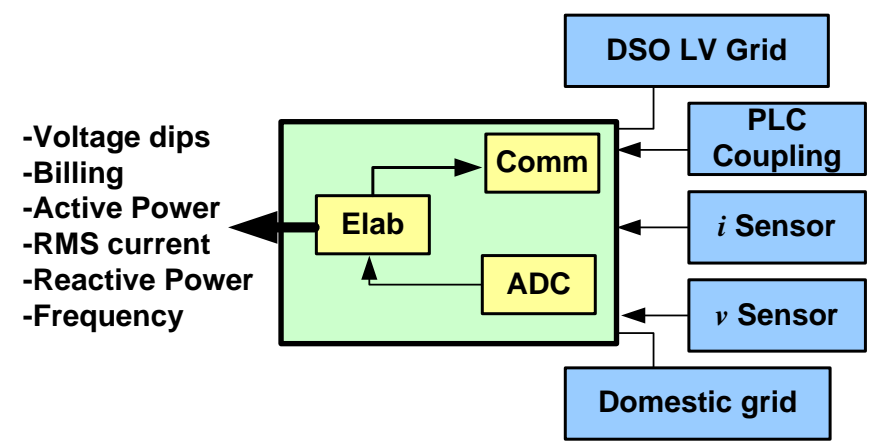

Fig. 5. Technique presented by Dede et al. [24]

The performance of the smart meter can be also enhanced if the readings of smart meter can be subjected for analysis. It leverages innovation in both services and process. Advance analytical operations significantly assist in understanding the hidden traits of usage data that further helps in understanding actual regulatory requirements. Study in such direction was carried out by Flath et al. [25] who have carried out cluster analysis on the usage data of smart meter considering different customer profiles further segregated to a day and week profiles. Both the profiles are further clustered with respect to season's summer, winter and its corresponding impact on week days and weekends. The study significantly contributes to integrate cluster analysis with business intelligence to further alleviate the performance. Nearly similar type of study was also carried out by Gajowniczek and Zabkowski [26] where the authors has used machine learning technique to perform forecasting of smart metered data. The study outcome was evaluated with respect to mean squared error and accuracy on the hourlybased data. Performance of smart meter could be also enhanced using optimization techniques. Hao et al. [27] has presented a technique that assists in identifying the original states of the electrical appliances' deploying and reduced quantity of smart meters. A tree network is designed to replicate the power distribution line and a unique optimization technique. The better performance of the smart meter could be also ensured by testing mechanism too. Janiga et al. [28] have discussed a testing mechanism that can evaluate the electrical parameters. However, such testing mechanism couldn't ensure much analysis of quality of power for low voltage networks. Work addressing such problem was carried out by Sanduleac et al. [29] where a method is presented to minimise the computational power of the smart meter and thereby improve its performance. The author has carried out the hardware design of the smart meter using ARM processor and investigated the trends in harmonic currents. The technique also performs statistical analysis over voltage level to scale the performance effectiveness. Most recently, Wakeel et al. [30] have presented a study where a conventional clustering technique $\mathrm{k}$-means algorithm is used for estimating load from the readings of domestic smart meter. The cluster analysis was found to provide significant enhancement to the load estimation. Panchadcharam et al. [31] have presented a simulation-based study to assess the time of transmission with different sizes of data. Most recently, Yang et al. [32] have presented a multiple access control based protocol developing a network of smart meters. Another important factor of performance efficiency is billing of the smart meters. There is 
less number of studies which focuses on minimizing the billing of usage as there are large number of complexities associated with cost of distributive generation. Hence, financial optimization becomes the sole factor for live billing process. Study in such direction was carried out by Zhang et al. [33]. The technique presented by the author exploits the electrical power exclusively for local distributed generators.

\section{B. Technqiues towards energy efficiency}

Study towards energy efficiency is meant for accomplishing an objective of energy control essentially. Usually, the technique calls for extracting the usage data by suppliers and then forwarding the processed and organised data to the consumer. Arif et al. [34] has presented a technique where a smart meter is designed using wireless networks. The author has used experimental approach where a new smart meter was designed using microcontroller and communication module has been developed using XBee and GSM modem. Hence, wireless network was used to forward to the user's handheld device and the usage data that is being captured by microcontroller. Govinda et al. [35] have also used microcontroller-based hardware design of smart meter as well as GSM modem to perform usage readings transmission.

Similar category of study was also carried out by Azasoo et al. [36]. However, here the authors have used design science research methodology over the prototype meter installed over Ghana city. The usage data from the meter is than transmitted using GPRS and PIC (Figure 6).

Studies for energy efficiency were also focused in the direction to achieve green ecosystem. Bera et al. [37] has developed a technique based on coalition game which performs aggregation of utility data from multiple smart meters and forward it to base station (Figure 7). The authors have also developed an energy consumption model with an objective function to minimise the cost of usage. The technique was simulated in NS-3 considering 50 smart meters and one base station. The study outcome was assessed with respect to energy consumption and delay mainly.

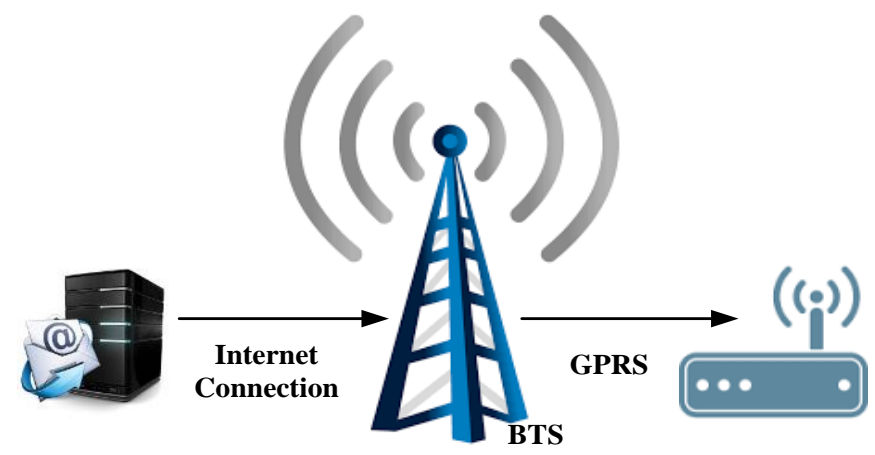

Fig. 6. Technique presented by Azasoo et al. [36]

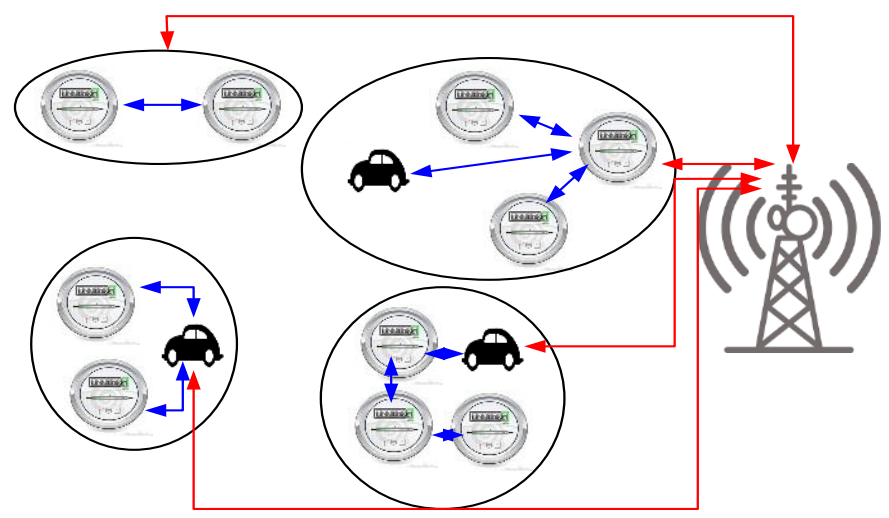

Fig. 7. Technique presented by Bera et al. [37]

Another study towards energy efficiency was put forward by Buchmann et al. [38] who have presented an analytical modelling for re-identification of dissipated energy records identical to specific utility feature. The authors have presented an empirical modelling that uses linear and integer linear optimization technique. The analysis was carried out using database of smart meter reading with respect to standard deviation and weights.

\section{Technqiue towards security}

Although the analogue device of smart meters already has anti-tampering circuitry unit, but still existing system is not enough to identify the compromised smart meters [39]. Moreover, the extracted utility data travels through a network whose security is still a matter of concern [40]. One of such research work has been carried out by Baig et al. [41]. The authors claimed that as the smart meters are connected to higher end networks (like internet), it is highly prone for vulnerable situations. The authors have presented a unique attack model that aims to generate erroneous meter readings. Finally, the authors have used message authentication mechanism to identify the attacker. The outcome of the study was assessed with respect to rate of attack detection with increasing score of compromised meters. Studies towards privacy preservation were carried out by Borges and Mulhhauser [42] for securing the communication system of smart meter. The technique also allows performing ciphering the aggregated data followed by decryption. A cryptographic approach was presented for this purpose. Studies towards enhancing privacy were also carried out by Jawurek et al. [43]. The technique allows preserving privacy along with billing related features using computational model programmed in Java. Kumar and Hussain [44] have implemented a simple cryptographic technique using message authentication principle. The entire operation of authentication is carried out with respect to secret key exchange using RSA, SHA256, and AES protocol. Similar study was also carried out by Agarwal et al. [45]. Qu et al. [46] has presented a technique to conserve the privacy using signature-based approach in order to conserve identity for resisting forgery attack. Sankar et al. [47] has presented a hypothetical framework in order to retain privacy of the smart meter. A framework is designed using hidden Markov model abstracting both the requirements of utility and privacy together. The model disclosed the facts that 
there is potential relationship among frequency components with low/high power components in presence of noise, which gives a pattern of privacy factor in security. The study outcome was testified with respect to auto-correlation, power spectral density,

Although cryptographic technique is used to perform encryption, which is again not $100 \%$ full-proof, if the utility data falls in wrong hand there is another level of new vulnerability i.e. understanding the current status of occupancy in any premises. It is quite understood that a house with people will have higher energy usage compared to house with less (or no) people in it. This information can be trapped in readings of smart meters and as such readings transmit over wire line or wireless network, its integrity is questionable. Hence, study in this problem was addressed by Chen et al. [48] most recently, where the authors has introduced a technique to resist detection of occupancy from readings of smart meter. According to this technique, a water heater (which is normally present in all houses) is autonomously switched on in a very controlled manner to give an illusion that there is someone in the house. The author has developed such water heater of 50 gallon with its explicit energy regulation. The study outcome was testified with respect to true and false positive / negative scores and was found to be better than other clustering techniques. Hence, adding certain external component assists in retaining the security was evident in various research works. Similar cases were found in Germany where Detken et al. [49] has introduced a grid that is resilient against tamper and was integrated with the hardware model. The authors have designed a core integrated with WLAN in order resist threat. Digital signature is the prime key to security in this work. Figure 8 shows the core security architecture used. The system uses TND (Trusted Network Device) to perform verification of the hardware and software elements in smart meters. The system also uses Trusted Platform Module (TPM) responsible for evaluating the trust factor. The system has also used LLDP (Link Layer Discovery Protocol) for exploring nodes (meters) in the neighbourhood.

Jafary et al. [50] have presented a study towards secure data forwarding mechanism from customer premise to the suppliers over distributed network. The technique uses minimal voltage communication of data using DLMS server to client over secure protocol. Sha et al. [51] have developed a technique of authentication using one-time password mechanism. The author has used asymmetric key to provide security against various lethal threats.

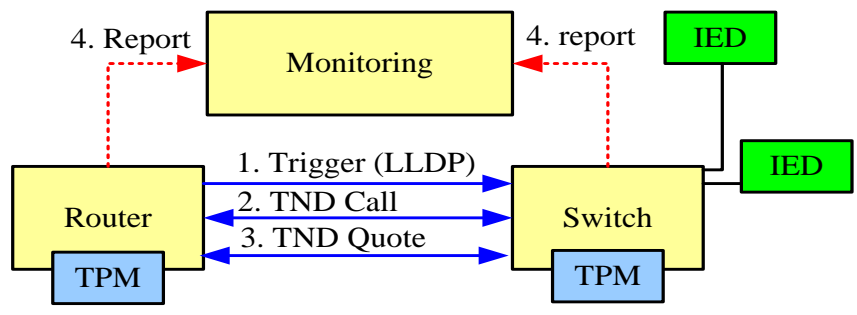

Fig. 8. Technique presented by Detken et al. [49]

TABLE I. SUMMARY OF EXISTING TECHNIQUES

\begin{tabular}{|c|c|c|c|c|}
\hline Authors & Problems & Techniques & Advantages & Limitation \\
\hline Alimardani et al. [21][22] & Non-synchronous signals & $\begin{array}{l}\text { Empirical approach with error } \\
\text { variance }\end{array}$ & $\begin{array}{l}\text { Better load distribution, can } \\
\text { estimate state on grids }\end{array}$ & $\begin{array}{l}\text {-Doesn't address energy control } \\
\text { problem. } \\
\text {-No comparative analysis }\end{array}$ \\
\hline Ciuciu et al. [23] & $\begin{array}{l}\text { Highly integrated network } \\
\text { for exchange of metered } \\
\text { data }\end{array}$ & $\begin{array}{l}\text { Context-based, Service } \\
\text { oriented Architecture }\end{array}$ & Innovative approach & $\begin{array}{l}\text {-Theoretical model without any } \\
\text { analysis }\end{array}$ \\
\hline Dede et al. [24] & Power management issue & $\begin{array}{l}\text { Network of smart meter } \\
\text { studied as Sensor network }\end{array}$ & Better data management & $\begin{array}{l}\text {-Doesn't consider transmission } \\
\text { complexity. -No comparative } \\
\text { analysis }\end{array}$ \\
\hline $\begin{array}{l}\text { Flath et al. [25] } \\
\text { Wakeel et al. [30] }\end{array}$ & $\begin{array}{l}\text { Consumption behaviour of } \\
\text { customer }\end{array}$ & Cluster Analysis & $\begin{array}{l}\text { Easily integrated with } \\
\text { business intelligence }\end{array}$ & $\begin{array}{l}\text {-No comparative analysis } \\
\text {-Accuracy not determined }\end{array}$ \\
\hline $\begin{array}{l}\text { Gajowniczek and } \\
\text { Zabkowski [26] }\end{array}$ & Forecasting of metered data & $\begin{array}{l}\text { Support vector machine } \\
\text { Neural Network }\end{array}$ & Simple predictive model & $\begin{array}{l}\text {-No comparative analysis } \\
\text {-non-linear optimization not } \\
\text { solved }\end{array}$ \\
\hline Hao et al. [27] & Deployment optimization & $\begin{array}{l}\text { Tree network, Greedy } \\
\text { Approach }\end{array}$ & Minimise smart meters & -No comparative analysis \\
\hline Janiga et al. [28] & Testing system & Experimental, LabView & Simple testing environment & -No comparative analysis \\
\hline Sanduleac et al. [29] & Evaluating power quality & $\begin{array}{l}\text { Experimental, Statistical } \\
\text { analysis, ARM processor }\end{array}$ & $\begin{array}{l}\text { Easier assessment of power } \\
\text { quality }\end{array}$ & $\begin{array}{l}\text {-No comparative analysis. } \\
\text {-No complexity analysis. }\end{array}$ \\
\hline Panchadcharam et al. [31] & Performance evaluation & $\begin{array}{l}\text { Simulation-based } \\
\text { infrastructure }\end{array}$ & $\begin{array}{l}\text { Effective realization of } \\
\text { transmission time }\end{array}$ & -No comparative analysis \\
\hline Yang et al. [32] & Performance enhancement & MAC protocol & $\begin{array}{l}\text { Maintains fairness, } \\
\text { scalability, delay }\end{array}$ & -No comparative analysis \\
\hline Arif et al. [34] & Energy efficiency & $\begin{array}{l}\text { Experimental, } \\
\text { Microcontroller, XBee and } \\
\text { GSM modem }\end{array}$ & $\begin{array}{l}\text { User friendly, less human } \\
\text { intervention, wireless }\end{array}$ & $\begin{array}{l}\text {-No comparative analysis } \\
\text {-Complexities on Wireless } \\
\text { network not considered. }\end{array}$ \\
\hline Govinda et al. [35] & Energy Efficiency & $\begin{array}{l}\text { Experimental, } \\
\text { Microcontroller, GSM } \\
\text { modem }\end{array}$ & User friendly & $\begin{array}{l}\text {-No comparative analysis } \\
\text {-Complexities on Wireless } \\
\text { network not considered. }\end{array}$ \\
\hline Azasoo et al. [36] & Minimise cost of billing & $\begin{array}{l}\text { Experimental, design science } \\
\text { research methodology, GPRS, } \\
\text { PIC }\end{array}$ & Minimised deployment cost & $\begin{array}{l}\text {-No comparative analysis } \\
\text {-Usage of GPRS is quite primitive } \\
\text { when } 5 \mathrm{G} \text { is about to launch }\end{array}$ \\
\hline Bera et al. [37] & Energy Efficiency & Game Theory (Coalition & Better QoS outcomes & -No comparative analysis \\
\hline
\end{tabular}




\begin{tabular}{|c|c|c|c|c|}
\hline & & game) & (energy, delay, lifetime, etc.) & $\begin{array}{l}\text {-algorithm complexity not } \\
\text { measured. }\end{array}$ \\
\hline Buchmann et al. [38] & Energy Efficiency & $\begin{array}{l}\text { Re-identification, analytical } \\
\text { framework }\end{array}$ & $\begin{array}{l}\text { Features assist in better } \\
\text { visualization to energy } \\
\text { consumption }\end{array}$ & $\begin{array}{l}\text {-No comparative analysis } \\
\text {-Doesn't address energy efficiency }\end{array}$ \\
\hline Baig et al. [41] & $\begin{array}{l}\text { Identifying compromised } \\
\text { meter }\end{array}$ & Message authentication & Better detection rate & $\begin{array}{l}\text {-No comparative analysis } \\
\text {-algorithm complexity not } \\
\text { measured. } \\
\text {-non-resistive against lethal threats }\end{array}$ \\
\hline $\begin{array}{l}\text { Borges and Mulhhauser } \\
\text { [42] }\end{array}$ & $\begin{array}{l}\text { Security in Smart Meter } \\
\text { Communication }\end{array}$ & Privacy preservation & Scalable, faster response & $\begin{array}{l}\text {-No comparative analysis } \\
\text {-Doesn't address energy efficiency }\end{array}$ \\
\hline Jawurek et al. [43] & Security & Privacy preservation & $\begin{array}{l}\text { Balances security and billing } \\
\text { system }\end{array}$ & $\begin{array}{l}\text {-Energy efficiency not addressed. } \\
\text {-No comparative analysis }\end{array}$ \\
\hline $\begin{array}{l}\text { Kumar and Hussain [44], } \\
\text { Agarwal et al. [45] }\end{array}$ & Security & RSA, AES, SHA256 & Good response time & $\begin{array}{l}\text {-RSA has bigger key size } \\
\text {-computational complexity is high. } \\
\text {-Energy efficiency not addressed. } \\
\text {-No comparative analysis }\end{array}$ \\
\hline Qu et al. [46] & Anonymity of usage data & $\begin{array}{l}\text { Anonymous credential } \\
\text { mechanism }\end{array}$ & Maintain data anonymity & $\begin{array}{l}\text {-No comparative analysis. } \\
\text {-No complexity analysis. } \\
\text {-Narrowed scope of attack } \\
\text { resistance }\end{array}$ \\
\hline Sankar et al. [47] & Privacy problem & $\begin{array}{l}\text { Hypothetical framework, } \\
\text { hidden Markov model }\end{array}$ & Better spectral efficiency, & -No comparative analysis \\
\hline Chen et al. [48] & $\begin{array}{l}\text { Resisting identification of } \\
\text { Occupancy }\end{array}$ & $\begin{array}{l}\text { Water heater regulating } \\
\text { energy usage }\end{array}$ & Better prevention technique & $\begin{array}{l}\text {-Leads to extra energy } \\
\text { consumption }\end{array}$ \\
\hline Detken et al. [49] & Security in smart grid & Trusted core network & $\begin{array}{l}\text { Sophisticated design for } \\
\text { security }\end{array}$ & $\begin{array}{l}\text {-Doesn't address energy efficiency } \\
\text {-No comparative analysis } \\
\text {-works only on low-level devices }\end{array}$ \\
\hline Jafary et al. [50] & Secure communication & Experimental approach & $\begin{array}{l}\text { Supports low voltage } \\
\text { communication }\end{array}$ & -No comparative analysis \\
\hline Sha et al. [51] & Authentication problem & $\begin{array}{l}\text { One-time password, } \\
\text { symmetric key }\end{array}$ & $\begin{array}{l}\text { Simple authentication } \\
\text { protocol }\end{array}$ & -Doesn't support energy efficiency \\
\hline
\end{tabular}

\section{RESEARCH GAP}

The prior section discusses about all the updated techniques found to be introduced in the area of smart meters. All the techniques are found to possess significant amount of advantages, features as well as limitations. However, this part of the section will discuss only those problems which were kept aloof the investigational area. This section presents the research gap explored after reviewing the prior literatures.

- Few studies on energy efficiency: At present, majority of the research work focuses on using smart meters that extracts the usage data and uses some sort of communication medium to transfer the usage data back to the customer. However, smart meters are not found to minimise energy usage autonomously. There are very less studies to prove that there is energy conservation after adopting the presented technique.

- Less study towards mathematical modelling: At present, the work being carried out uses empirical modelling, analytical modelling, and experimental modelling. Lack of mathematical modelling in the area of controlling mechanism is one of the significant research gaps. Although, some computational model exists, but they lack validity as well as computational complexity analysis.

- Fewer studies towards uncertainty handling: There are many real-time scenarios where various forms of uncertain features are less emphasised. Presence of uncertainties usually occurs from the transmission process as well as raw data collection process. Although, there are some works being done in predictive approach, but they do not utilise uncertainty modelling that required joint implementation of timeseries, stochastic, and probability theory. There is a need of technique which can perform optimization in presence of less input or vague inputs to the optimization algorithm.

- Few Benchmarked Study: At present, 99\% of the research manuscript towards solving the problems of smart meters doesn't use performance comparative analysis. This makes it quite hard to understand the best work till date on a specific problem.

\section{POSSIBLE LINE OF RESEARCH}

Smart meters are not only meant for automating the billing generation process but also should have exclusive feature to minimise the energy consumption. The possible line of research could be in the direction of overcoming the research gap presented in prior section:

- Novel mathematical framework for energy efficiency: A mathematical framework could be designed that can perform modelling of energy based on the usage data from smart meters. There is also a need to develop a novel integer linear optimization algorithm to consider mapping the uncertainties over the distributive generation process. All this mechanism can be used for developing a new controlling mechanism. The mathematical model should be also subjected to convergence test to understand its effectiveness as well as its applicability towards energy efficiency. The best way to evaluate the outcome will be to look for down pattern of pricing of electricity over increasing time of usage. 
- Novel framework for robust wireless transmission: The existing literatures towards wireless transmission don't consider various complexities present in wireless environment. Hence, a framework could be developed which can use concept of decision making, stochastic, probability theory in order to handle the uncertainty condition over wireless transmission (of reading of smart meter). Various constraint factors e.g. throughput, latency, heterogeneity, etc. should also be considered while modelling. Fault tolerant performance could be measured by increased utility over smart grid.

- Novel framework for behaviour analysis: The existing security technique uses cryptographic technique to resist attacks or compromising the readings of smart meter. Such techniques are quite symptomatic. As futuristic readings of smart meters could be transmitted via cloud, than it is further more exposed to vulnerability. Hence, it is essential to understand the unpredictable behaviour of the vulnerable situation so that it (readings) could be securely routed via any medium. Hence, a novel framework could be designed for this purpose.

\section{CONCLUSION}

The overall goal of this paper is to illustrate the existing approaches used for enhancing the performance of smart metering system. This manuscript discusses about the smart meter with an aid of its essential components, basic operation, existing research trends, exploring research gap, followed by anticipated line of research work in future. The studies show that there are various forms of techniques being used for addressing different ranges of problems associated with smart metering system. The prime motivation to work on smart meter is due to its balanced advantages to both consumer and utilities e.g. i) transparency of usage information and billing related information, ii) Maximised information about the service delivery, iii) generates awareness among consumers to save energy, iv) minimises outage conditions and demand peaks, v) faster process of monitoring electrical system with dynamic pricing and many more. However, after reviewing the various scripted literatures, it is found that there are various impediments toward research work in smart meters e.g. i) expensive affair as there is a need of transition from old to new technology, ii) more exposed to security risk especially the privacy factor. The prime contribution of this review paper is its findings associated with the effectiveness of existing system i.e. i) less studies are found to be benchmarked, ii) more adoption of experimental approach compared to computational modelling in real sensor, iii) less focus on investigating how wireless technologies improves energy efficiency, etc. Hence, the future work will be in a direction to cover up the above mentioned issues.

\section{REFERENCES}

[1] T. Wojcicki, "VLSI: Circuits for Emerging Applications", CRC Press, 2014

[2] A. Faruqui, K. Eakin, "Electricity Pricing in Transition", Springer Science \& Business Media, 2012

[3] J. Ekanayake, N. Jenkins, K. Liyanage, J. Wu, A. Yokoyama, "Smart Grid: Technology and Applications", John Wiley \& Sons, 2012

[4] F. Toledo, "Smart Metering Handbook", PennWell Books, 2013
[5] F. Behmann, K. Wu, "Collaborative Internet of Things (C-IoT): for Future Smart Connected Life and Business", John Wiley \& Sons, 2015

[6] G. Fortino, P. Trunfio, "Internet of Things Based on Smart Objects: Technology, Middleware and Applications", Springer Science \& Business Media, 2014

[7] A B M Shawkat Ali, "Smart Grids: Opportunities, Developments, and Trends", Springer Science \& Business Media, 2013

[8] "Get

Smarter.Faster", https://www.maximintegrated.com/en/landing/index.mvp?lpk=634, Retrived, 03th Jan, 2017

[9] "Smarter Electricity Meters", https://www.maximintegrated.com/en/solutions/smart-electricitymeters/index.mvp?CMP =selsoln, Retrived, 3rd Jan, 2017

[10] "Texas Instruments, Implementation of a Three-Phase Electronic WattHour Meter Using the MSP430F471xx", Application Report \#SLAA409A, 2009

[11] "Energy Metering ICs", http://www.analog.com/en/products/analog-todigital-converters/integrated-special-purpose-converters/energymetering-ics.html, Retrived, 3rd 2017

[12] T. Cooke, "Power Quality Measurement Capabilities in Smart Revenue Meters", Report from Electric power Research Institute, 2014

[13] J. Zheng, D. W. Gao, L. Lin, "Smart Meters in Smart Grid: An Overview", IEEE Green Technologies Conference, pp.57-64, 2013

[14] A. H. Rosenfeld, D. A. Bulleit, and R. A. Peddie, "Smart Meters and Spot Pricing: Experiments and Potential", IEEE Technology and Society Magazine, vol.5, Iss.1, 1986

[15] I. Opris, L. Caracasian, "The relation between smart meters and electricity consumers", IEEE International Conference on Environment and Electrical Engineering, pp.325-329, 2013

[16] H. L. M. do Amaral, A. N. de Souza, D. S. Gastaldello, F. Fernandes, "Smart meters as a tool for energy efficiency" IEEE International Conference on Industrial Application, pp.1-6, 2014

[17] Q. Sun, H. Li, Z. Ma, C. Wang, J. Campillo, Q. Zhang, F. Wallin, J. Guo, "A Comprehensive Review of Smart Energy Meters in Intelligent Energy Networks", IEEE Internet of Things Journal, vol.3, iss.4, pp.464-479, 2015

[18] N. S. Zivic, O. Ur-Rehman, and C. Ruland, "Evolution of Smart Metering Systems", IEEE-23rd Telecommunications forum, pp.635-638, 2015

[19] D. Alahakoon, X. Yu, "Smart Electricity Meter Data Intelligence for Future Energy Systems: A Survey", IEEE Transactions on Industrial Informatics, vol.12, iss.1, pp.425-436, 2015

[20] J. Zheng, L. Lin, D. W. Gao, "Smart Meters in Smart Grid: An Overview”, IEEE Green Technologies Conference, pp.57-64, 2013

[21] A. Alimardani, S. Zadkhast, J. Jatskevich, "Using Smart Meters in State Estimation of Distribution Networks", IEEE Conference and Exposition, pp.1-5, 2014

[22] A. Alimardani, F. Therrien, D. Atanackovic, J. Jatskevich, "Distribution System State Estimation Based on Nonsynchronized Smart Meters", IEEE Transactions on Smart Grid, vol.6, Iss.6, pp.2919-2928, 2015

[23] I. G. Ciuciu, R. Meersman, T. Dillon, "Social Network of SmartMetered Homes and SMEs for Grid-based Renewable Energy Exchange", IEEE International Conference on Digital Ecosystems and Technologies, pp.1-6, 2012

[24] A. Dede, D. D. Giustina, S. Rinaldi, P. Ferrari, A. Flammini, A. Vezzoli, "Smart Meters as Part of a Sensor Network for Monitoring the Low Voltage Grid”, IEEE Sensor Applications Symposium, pp.1-6, 2015

[25] C. Flath, D. Nicolay, T. Conte, "Cluster Analysis of SmartMetering Data", Bise-Research Paper. Gabler Verlag, vol.1, pp.31-39, 2012

[26] K. Gajowniczeka, T. Ząbkowskia, "Short term electricity forecasting using individual smart meter data", Elsevier-ScienceDirect, International Conference on Knowledge-Based and Intelligent, Information \& Engineering Systems, vol.35, pp.589-597, 2014

[27] X. Hao, Y. Wang, C. Wu, "Smart Meter Deployment Optimization for Efficient Electrical Appliance State Monitoring", IEEE Third International Conference on Smart Grid Communication, pp.25-30, 2012 
[28] P. Janiga, M. Liska, V. Volcko, B. Pilat, "Testing system for smart meters", IEEE International Scientific Conference on Electric Power Engineering, pp.519-522, 2015

[29] M. Sanduleac, M. Albu, J. Martins, M. D. Alacreu, C. Stanescu, "Power Quality Assessment in LV networks using new Smart Meters design", IEEE International Conference on Compatibility and power electronics, pp.106-112, 2015

[30] A. Al-Wakeel, J. Wu, N. Jenkins, "k-means based load estimation of domestic smart meter measurements", Elsevier-Applied Energy, 2016

[31] S. Panchadcharam, G. A. Taylor, Q. Ni, I. Pisica, S. Fateri, "Performance Evaluation of Smart Metering Infrastructure using Simulation Tool", IEEE International Universities power Engineering Conference, pp.1-6, 2012

[32] Y. Yang, Y. Yin, Z. Hu, "MAC Protocols Design for Smart Metering Network", Science Publishing Group-Automation, Control and Intelligent Systems, vol.3, Iss.5, pp.87-94, 2015

[33] H. Zhang, D. Zhao, C. Gu, F. Li, B. Wang, "Economic optimization of smart distribution networks considering real-time pricing", Springer Journal of Modern Power System and Clean Energy, vol.2, Iss.4, pp.350-356, 2014

[34] A. Arif, M. AI-Hussain, N. AI-Mutairi, E. AI-Ammar, "Experimental Study and Design of Smart Energy Meter for the Smart Grid", IEEE International renewable and sustainance energy conference, pp.515520,2013

[35] Govinda.K, "Design of Smart Meter using Atmel 89S52 Microcontroller", Elsevier-ScienceDirect, vol.21, pp.376-380, 2015

[36] J. Q. Azasoo, K. O. Boateng, "A Retrofit Design Science Methodology for Smart Metering Design in Developing Countries", IEEEInternational Conference on Computational Science and Its Applications, pp.1-7, 2015

[37] S. Bera, S. Misra, M. S. Obaidat, "Energy-Efficient Smart Metering for Green Smart Grid Communication", IEEE global Communications Conference, pp.2466-2471, 2014

[38] E. Buchmann, K. Bohm, T. Burghardt, S. Kessler, "Re-identification of Smart Meter data", Springer Personal Ubiquitous Computing, vol.17, pp.653-662, 2013

[39] O. Ur-Rehman, N. Zivic, C. Ruland, "Security Issues in Smart Metering Systems", IEEE International Conference on Smart Energy Grid Engineering, pp.1-7, 2015
[40] R. Mahmud, R. Vallakati, A. Mukherjee, "A Survey on Smart Grid Metering Infrastructures: Threats and Solutions", IEEE International Conference on Electro/Information Technology, pp.386-391, 2015

[41] Z. A. Baig, A. Al Amoudy, K. Salah, "Detection of Compromised Smart Meters in the Advanced Metering Infrastructure", Proceedings Of The 8th IEEE GCC Conference And Exhibition, Muscat, Oman, 2015

[42] F. Borges, M. Muhlhauser, "EPPP4SMS: Efficient Privacy-Preserving Protocol for Smart Metering Systems and Its Simulation Using RealWorld Data", IEEE Transactions on Smart Grid, vol.5, No.6, 2014

[43] M. Jawurek, M. Johns, and F. Kerschbaum, "Plug-In Privacy for Smart Metering Billing", IEEE Transactions on Smart Grid, vol.5, iss.6, pp.2701-2708, 2014

[44] V. Kumar and M. Hussain, "Secure communication for advance metering infrastructure in smart grid", Annual IEEE India Conference, pp.1-6, 2014

[45] S. Agarwal, A. Kumar, C. Fatnani, "An Intelligent Smart Metering System For AMI With Efficient Electrical Appliance State Monitoring", Proceedings of IEEE TechSym 2014 Satellite Conference, 2014

[46] H. Qu, P. Shang, X-J Lin, and L. Sun, "Cryptanalysis of A PrivacyPreserving Smart Metering Scheme Using Linkable Anonymous Credential", IACR Cryptology ePrint Archive, 2015

[47] L. Sankar, S. R. Rajagopalan, S. Mohajer, "Smart Meter Privacy: A Theoretical Framework", IEEE Transactions on Smart Grid, vol.4, iss.2, pp.837-846, 2013

[48] D. Chen, S. Kalra, D. Irwin, "Preventing Occupancy Detection From Smart Meters", IEEE Transactions On Smart Grid, vol.6, iss.6, pp.24262434,2015

[49] K-O Detken, C-H Genzel, C. Rudolph, M. Jahnke, "Integrity Protection in a Smart Grid Environment for Wireless Access of Smart Meters", IEEE International Symposium on Wireless Systems, Conferences on Intelligent Data Acquisition and Advanced Computing Systems, 2014

[50] P. Jafary, S. Repo, H. Koivisto, "Secure Communication of Smart Metering Data in the Smart Grid Secondary Substation", IEEE Innovative Smart Grid Technologies, pp.1-6, 2015

[51] K. Sha, C. Xu, Z. Wang, "One-time Symmetric Key Based Cloud Supported Secure Smart Meter Reading", IEEE International Conference on Computer Communication and Networks, pp.1-6, 2014 